\title{
Item-specific and generalization effects on brain activation when learning Chinese characters
}

\author{
Yuan Deng a,b, James R. Booth ${ }^{\mathrm{c}}$, Tai-Li Chou ${ }^{\mathrm{c}, \mathrm{d}}$, Guo-Sheng Ding ${ }^{\mathrm{a}}$, Dan-Ling Peng ${ }^{\mathrm{a}, *}$ \\ ${ }^{a}$ State Key Lab of Cognitive Neuroscience and Learning, Beijing Normal University, Beijing 100875, PR China \\ ${ }^{\mathrm{b}}$ Institute of Psychology, Chinese Academy of Science, Beijing 100101, PR China \\ ${ }^{\mathrm{c}}$ Department of Communication Sciences and Disorders, Northwestern University, Evanston, IL 60208, USA \\ ${ }^{\mathrm{d}}$ Department of Psychology, National Taiwan University, Taipei 10617, Taiwan \\ Received 18 May 2007; received in revised form 16 August 2007; accepted 9 September 2007 \\ Available online 22 September 2007
}

\begin{abstract}
Neural changes related to learning of the meaning of Chinese characters in English speakers were examined using functional magnetic resonance imaging (fMRI). We examined item specific learning effects for trained characters, but also the generalization of semantic knowledge to novel transfer characters that shared a semantic radical (part of a character that gives a clue to word meaning, e.g. water for lake) with trained characters. Behavioral results show that acquired semantic knowledge improves performance for both trained and transfer characters. Neuroimaging results show that the left fusiform gyrus plays a central role in the visual processing of orthographic information in characters. The left superior parietal cortex seems to play a crucial role in learning the visual-spatial aspects of the characters because it shows learning related decreases for trained characters, is correlated with behavioral improvement from early to late in learning for the trained characters, and is correlated with better longterm retention for the transfer characters. The inferior frontal gyrus seems to be associated with the efficiency of retrieving and manipulating semantic representations because there are learning related decreases for trained characters and this decrease is correlated with greater behavioral improvement from early to late in learning.
\end{abstract}

C) 2007 Published by Elsevier Ltd.

Keywords: Lexical; Training; Transfer; fMRI; Chinese; Radical

\section{Introduction}

Lexical learning/training studies provide a dynamic method for evaluating the neural network involved in language processing. These studies have suggested that left fusiform is involved in new orthographic word form or object form learning (Gauthier, Tarr, Anderson, Skudlarski, \& Gore, 1999; Sandak et al., 2004; Xue, Chen, Jin, \& Dong, 2006), the superior temporal gyrus is involved in learning phonological representations (Callan et al., 2003; Wang, Sereno, Jongman, \& Hirsch, 2003), the middle temporal gyrus is involved in learning semantic information (Sandak et al., 2004) and the inferior parietal cortex is involved in the mapping between orthographic word forms and their phonological representations (Eden et al., 2004; Hashimoto \& Sakai, 2004; Lee et al., 2003). Other findings, including connectivity

\footnotetext{
* Corresponding author. Tel.: +86 10 58808939; fax: +86 1058808939.

E-mail address: pd13507@bnu.edu.cn (D.-L. Peng).
}

analysis, have also suggested a shift from an initial rule-based process in the temporo-parietal region to a later pattern-based process in the occipito-temporal region with training (Buchel, Coull, \& Friston, 1999; Hashimoto \& Sakai, 2004; Poldrack, Desmond, Glover, \& Gabrieli, 1998).

Acquiring lexical knowledge is marked by the acquisition of rules of the language system, even without explicit instructions. One way to explore the effect of current knowledge on processing new information is to compare the brain activity on trained words with that on novel words. Several studies have reported similar pattern of activation for trained words and for novel words (Callan et al., 2005; Hashimoto \& Sakai, 2004; Lee et al., 2003) probably due to the high overlap in lexical features between novel words and trained ones. In contrast to this, Sandak et al. (2004) found that the activation pattern on trained words showed the highest degree of similarity with that of real words, whereas the least similarity was shown between novel and real words. However, other studies have not showed activity changes over training on novel words (Breitenstein et 
al., 2005; Stein et al., 2006). It is necessary to note that the generalization of learned knowledge could be based on perceptual features (such as visual similarities) or rules (such as syntax). However, the design of previous studies has not allowed a disassociation of feature-based from rule-based transfer. Although these studies compared brain activation for trained words with that for novel words, they could not examine the application of abstract knowledge or rules to the processing of novel words.

Chinese phonograms offer a unique tool for exploring the transfer of lexical knowledge. Phonograms, which account for 80-90\% of characters in modern Chinese, are generally composed of two components called radicals: one gives a clue to word meaning, e.g. water for lake, and the other indicates pronunciation. This unique characteristic of the Chinese phonogram makes it possible to extract underlying lexical knowledge during learning. When several semantically related words have the same semantic radical, the semantic radical gives a clue to word meaning. For example, the three words '海' (sea), ‘湖' (lake) and '河' (river) all have the same semantic radical '; ' on their left part, which indicates something related to 'water'. In the current study, learning this knowledge was expected to facilitate the character recognition. Given that this sub-lexical component gives a clue to word meaning, we could examine transfer of this knowledge to novel characters that shared the same semantic radical with trained characters, such as the new character 溪 ' (brook) with the semantic radical ' $;$ ' (water). By comparing the learning effect of characters that shared a semantic radical to those that did not share a semantic radical, the similarity of visual features across characters was controlled and therefore any transfer effects on novel characters should be due to abstract linguistic knowledge.

Another advantage of using Chinese characters in English learners is that it allows an exploration of language-specific activation. In previous studies, participants were trained on a language with an orthographic system similar to their native language (Hashimoto \& Sakai, 2004; Lee et al., 2003; Raboyeau et al., 2004), or English speakers learned artificial languages written in alphabetic scripts (Breitenstein et al., 2005; Sandak et al., 2003). These studies did not allow an examination of whether network components are similar or different between languages with fundamentally different orthographic systems. No study has examined the learning-induced neural changes across alphabetic versus non-alphabetic orthographic systems, such as Chinese characters. Evidence from bilingual and monolingual imaging studies have suggested that Chinese and English share a similar semantic system (Bolger, Perfetti, \& Schneider, 2005; Booth et al., 2006; Chee, Tan, \& Thiel, 1999; Chee et al., 2000, 2003), even for nonfluent bilinguals (Xue, Dong, Jin, Zhang, \& Wang, 2004). However, there are still distinct neural mechanisms for Chinese character processing (Tan, Laird, Li, $\&$ Fox, 2005). More bilateral activation in the visual cortex has been shown for Chinese character reading, and some have suggested that this is due to the greater demands on visual-spatial analysis (Bolger et al., 2005; Tan et al., 2003). The left middle frontal gyrus (BA9) is often found activated in Chinese character reading, but rarely in alphabetic reading. Some have suggested that this area is related to the addressed (whole character) access of phonological representations in Chinese (Tan et al., 2000; Tan, Feng, Fox, \& Gao, 2001; Tan, Liu et al., 2001; Tan et al., 2005). Activation of bilateral precuneus and superior parietal lobule (SPL, BA7) are also consistently reported for Chinese character reading (Chee et al., 1999, 2000; Kuo et al., 2001, 2003, 2004; Lee et al., 2004; Tan et al., 2000; Tan, Feng et al., 2001; Tan, Liu et al., 2001). According to a meta-analysis study (Tan et al., 2005), the dorsal part of left parietal cortex showed consistent activation for phonological processing of Chinese characters, in contrast to activation of the ventral part for English processing.

In this study, English speakers with no previous exposure to non-alphabetic languages were asked to learn Chinese phonograms without explicit instruction on the meaning of semantic radical. The key purposes of this study were to examine the neural mechanism involved in (1) learning of Chinese characters in English speakers and (2) transfer of semantic knowledge to novel words. Previous studies have shown learning effects for visual information in the fusiform gyrus (Gauthier et al., 1999; Sandak et al., 2004) and a shift from parietal cortex to the fusiform gyrus (Poldrack et al., 1998). Because our training involved relatively few items over a relatively short amount of time, the learning effect could be pronounced in only in the parietal region. Previous research has also implicated the middle temporal gyrus in verbal semantic representations (Chou, Booth, Bitan et al., 2006; Chou, Booth, Burman et al., 2006). However, we expected little or no learning effects here because we trained English speakers on the English translations of Chinese characters, and we would not expect this to result in a reorganization of the verbal semantic system. Because responses to novel stimuli are likely to place greater demands on visual spatial processing (Dolan et al., 1997; Kuo et al., 2004) as well as greater demands on retrieval and selection processes (Clark \& Wagner, 2003; Corbetta \& Shulman, 2002), we expected to see greater activation in superior parietal cortex and inferior frontal gyrus, especially for transfer stimuli as compared to the trained stimuli.

\section{Methods}

\subsection{Participants}

Twelve adults (average age $=21.7$ years, range $=19-39$ years, 10 females and 2 males) participated in this study. All participants were undergraduate or graduate students at Northwestern University. Participants were native English speakers with no prior Chinese language and other non-alphabetic language experience. Participants were right-handed and had normal hearing and normal or correct-to-normal vision. None of the participants had a history of learning disability, attention deficit hyperactivity disorder (ADHD), psychiatric disorder, speech articulation problems or neurological disease. The Institutional Review Board at Northwestern University and Evanston Northwestern Healthcare Research Institute approved the informed consent procedures. All participants got monetary reimbursement for their participation.

\subsection{Materials}

One hundred and forty-four Chinese phonograms evenly consisted of two character types: semantic-implied (SI) versus semantic-unrelated (SU). For SI type, 12 groups of six exemplars shared the same semantic radical, which indicates the meaning/semantic category of the whole character. Within these groups, the phonetic radical of these characters varied from each other (Fig. 1a). For SU type, 12 groups contained six exemplars in which the semantic radicals were 


\section{Trained}

(a)

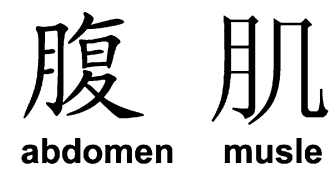

fu4

(b)

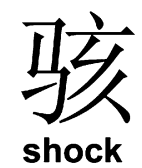

ji1

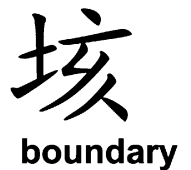

hai4

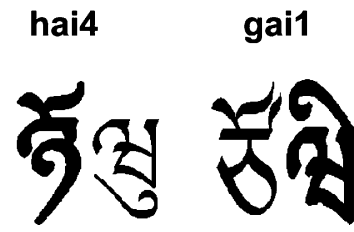

(c)

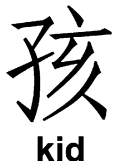

Transfer

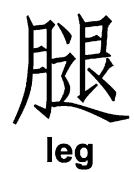

tui3

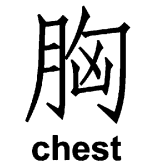

xiong1

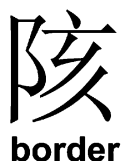

hai2 border

gai1

Fig. 1. Examples of stimuli with their English translation and their pronunciation in pinyin. Numbers for the pinyin indicate one of four tones: (a) semantic-implied type characters, the left part of each character (semantic radical) indicates "body part"; (b) semantic-unrelated type characters, the left part of each character indicates different meanings, while the right part (phonetic radical) of each character is the same; (c) two examples of visual stimulus for the baseline task in the fMRI scan with the left part or right part of the symbol bolded.

all different, so it was impossible to extract the semantic radical as a clue to the meaning. However, in order to balance the visual repetition as compared to SI type, the six exemplars in each group of the SU type shared the same phonetic radical (Fig. 1b). All characters were evenly divided into two stimuli sets. Each set included three exemplars of each radical of both character types; as a result, there were a total of 72 characters in each stimulus set. These two stimulus sets were counterbalanced for either training or transfer across participants, i.e. for one half of the participants, one stimulus set was used for training and the other for transfer; the reverse arrangement was applied to the other half of the participants. Characters used for training were broken up into six lists of 12 characters. Each list included two radicals (six characters) in SI type and another two radicals (six characters) in SU type.

All characters were about a $200 \times 200$ pixel size. The characters with the same radical were presented in three different fonts (SimSun, KaiTi and Fangsong) in order to encourage participants to perform the task based on the extraction of orthographic information rather than on the recognition of lowlevel visual similarity. The stroke count of characters, an indication of visual complexity, did not show significant differences between the two stimuli sets, six lists or two character types. Chinese characters in the SI type showed significantly higher written frequency (1485 per million) than those in the SU type (519 per million) (Wang, Chang, \& Li, 1985). However, because all participants had no prior experience with Chinese characters, this difference could not affect training or transfer effects. Both written and spoken frequency of English translations (data base: Baayen, Piepenbrock, \& Gulikers, 1995 did not show significant differences between stimuli sets, lists or character types. The free association value of the radical to the character meaning for the English translations (data base: Nelson, McEvoy, \& Schreiber, 1998) did not show significant differences between the stimulus sets or lists $($ mean $=0.14)$. Because the semantic radicals in the SU type were different, no free association values were collected for this type.

Each Chinese character was given a one-word English translation in meaning in an auditory format in order to avoid the visual interference of word forms between languages. All English translations were recorded in a soundproof booth using a digital recorder and a high-quality stereo microphone. A native Chicagoan woman read aloud each English translation in isolation so that there would be no contextual effects. Sound waves from the English translations were normalized to $800 \mathrm{~ms}$ and equal amplitude (loudness) (WaveLab, 2002).
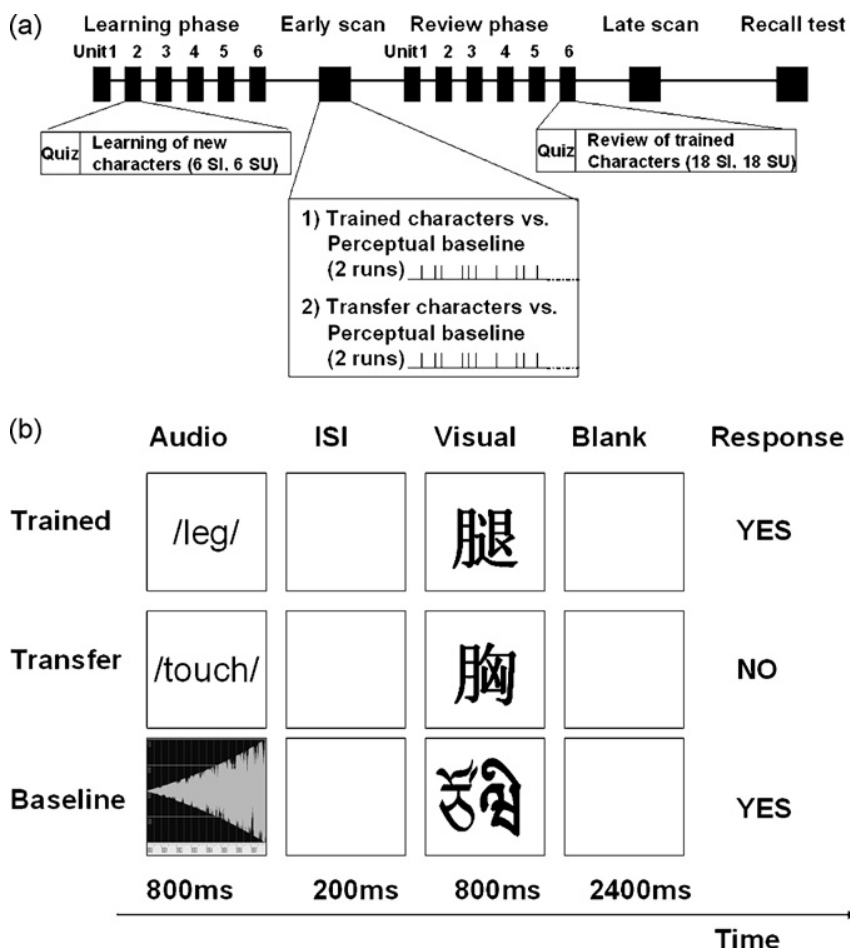

Fig. 2. (a) Illustration of overall training procedure; (b) illustration of experiment tasks in the fMRI session.

\subsection{Procedure}

\subsubsection{Behavioral training}

The overall training procedure is presented in Fig. 2. Every participant went through both a learning and a review phase. Regular quizzes were administered to assess the progress of participants. All participants were trained in a quiet isolated room. All training and testing procedures were computerized.

In the learning phase, participants learned a new list about every other day and finished all six lists within 2 weeks. The learning sequence of the six lists was counterbalanced between participants by a Latin square arrangement. Within each learning unit (i.e. day), participants saw a Chinese character on the center of a screen for $30 \mathrm{~s}$ and simultaneously heard its English translation in meaning repeat 6 times. Each character was presented 4 times so that the repetition of a specific radical was 12 times. The sequence in which the characters were presented was fixed in each learning unit, ensuring the three exemplars of a certain radical were not contiguously presented and a certain character was not contiguously repeated. Each learning unit took $24 \mathrm{~min}$. After finishing the six learning units, participants reviewed all trained characters in six review units of three lists each. The arrangement of review lists was designed ensuring that each list was reviewed three times on different days, and a particular list would not be reviewed more than twice on consecutive days. Because each participant started with a different learning list, the reviewing sequence also varied across participants. Within each review unit, participants saw a Chinese character on the center of a screen for $10 \mathrm{~s}$ and simultaneously heard its English translation in meaning repeat 3 times. Each character was repeated 4 times, resulting in the repetition of a certain radical 12 times. Characters were presented randomly with the constraint that a particular character did not appear contiguously more than twice. Each review unit lasted $24 \mathrm{~min}$.

The quiz was taken before learning or reviewing the list(s) on the participant's next visit in order to deal with immediacy effects. Each quiz only covered trained characters from the latest learning or review unit, i.e. no novel stimuli were introduced into quizzes. Within each trial, an auditory meaning was presented for $800 \mathrm{~ms}$, followed by a $200 \mathrm{~ms}$ interstimulus interval (ISI), then a visual character for $800 \mathrm{~ms}$. After a $2400 \mathrm{~ms}$ blank, the next pair appeared. Participants were asked to judge whether the pair matched or not. Feedback was given after each response. Reaction time was recorded and defined as the latency from the 
point that a visual character appeared to the time that a participant pressed the button. All pairings were presented randomly.

\subsection{2. fMRI sessions}

Participants were scanned at the end of the learning phase and review phase. Participants were tested on 72 trained characters and another 72 transfer characters. The same set of trained and transfer material was used in the early and late scan, in which each character was always paired with the same English word. The task format in the scan was the same as that in the quizzes, except no feedback was given after each response (Fig. 2b). For trained characters, participants were asked to judge whether the pair matched or not. For transfer characters, participants were instructed to guess whether the pair matched based on their learned knowledge.

A visual-auditory matching task was served as a baseline for all runs. In this task, artificial visual symbols were paired with two kinds of complex tones. In order to minimize perceptual differences, these visual symbols were created by spatially combining two to four Tibetan characters into a left-right structural character, as seen in Fig. 1c. The size of these symbols was the same as that of Chinese characters. Each visual symbol was bolded on the left or right. A volume-increasing tone indicated the bolded part should be on the right; whereas a volume-decreasing tone indicated the bolded part should be on the left. Participants were asked to judge whether the position of the bolded part of the symbol matched the part indicated by the complex tone (Fig. 2b). This task was similar to the lexical task in terms of perceptual and response demands except that stimuli in the control task were meaningless. Because the stimuli in the control task were novel, comparing the lexical task to this baseline allowed examination of neural activation specific to the acquired characters.

Participants were trained to keep their head still using an infrared tracking device and then they practiced the baseline task in a simulator scanner for $15 \mathrm{~min}$ in order to acclimate themselves to the scanner environment (Rosenberg et al., 1997) and the control task. The practice session was administrated at least 3 days (range from 3 to 7 days) before the first fMRI scan. During the fMRI scan, the presentation of trials was optimized for event-related design using Optseq (http://surfer.nmr.mgh.harvard.edu/optseq, written by D. Greve, Charlestown, MA). There were two runs for trained characters, including 90 SI type pairings, 90 SU type pairings, 60 control trails and 74 null events. Duration of each run was $11.84 \mathrm{~min}$. There were another two runs for transfer characters, involving 60 SI type pairings, $60 \mathrm{SU}$ type pairings, 60 control trails and 56 null events. Each run lasted $8.95 \mathrm{~min}$. Both early and late scan used the same scanning protocol.

\subsubsection{Delayed-test on recall: radical knowledge test}

In order to examine the retention effect on trained knowledge, 3-6 months after the training, participants were asked back to the lab for a recall test. After going through a regular review unit on the six training lists, participants were shown a list of trained characters, in which the semantic radical of each character was circled. They were asked to write down the meaning of each character, and also to guess the meaning of the circled part. The rate for correctly interpreting the semantic radical was treated as the score for radical knowledge, indicating the extent to which participants had extracted the underlying lexical rule.

\subsection{Image acquisition}

Participants lay in the scanner with their head position secured with a specially designed vacuum pillow (Bionix, Toledo, $\mathrm{OH}$ ). An optical response box (Current Designs, Philadelphia, PA) was placed in the participants' right hand. The head coil was positioned over the participants' head. Participants viewed visual stimuli that were projected onto a screen via a mirror attached to the inside of the head coil. Participants wore headphones to hear auditory stimuli (Resonance Technology, Northridge, CA).

All images were acquired using a $1.5 \mathrm{~T}$ GE scanner. Gradient-echo localizer images were acquired to determine the placement of the functional slices. For the functional imaging studies, a susceptibility weighted single-shot EPI (echo planar imaging) method with BOLD (blood oxygenation level-dependent) was used. Functional images were interleaved from bottom to top in a whole brain EPI acquisition. The following scan parameters were used: $\mathrm{TE}=35 \mathrm{~ms}$, flip angle $=90^{\circ}$, matrix size $=64 \times 64$, field of view $=24 \mathrm{~cm}$, slice thickness $=5 \mathrm{~mm}$, number of slices $=24, \mathrm{TR}=2000 \mathrm{~ms}$. The first two functional runs had 349 image volumes each and the last two had 236 image volumes. In addition, a high resolution, T1 weighted 3D image was acquired (SPGR, TR $=21 \mathrm{~ms}$, $\mathrm{TE}=8 \mathrm{~ms}$, flip angle $=20^{\circ}$, matrix size $=256 \times 256$, field of view $=22 \mathrm{~cm}$, slice thickness $=1 \mathrm{~mm}$, number of slices $=124$ ). The orientation of the 3D image was identical to the functional slices.

\section{5. fMRI data analysis}

Data analysis was performed using SPM2 (Statistical Parametric Mapping). The functional images were corrected for differences in slice-acquisition time to the middle volume and were realigned to the first volume in the scanning session using affine transformations. No participant had more than $3.0 \mathrm{~mm}$ of movement in any plane. Co-registered images were normalized to the Montreal Neurological Institute (MNI) average template (12 linear affine parameters for brain size and position, 8 nonlinear iterations and $2 \times 2 \times 2$ nonlinear basis functions). Statistical analyses were calculated on the smoothed data $(10 \mathrm{~mm}$ isotropic Gaussian kernel), with a high pass filter ( $128 \mathrm{~s}$ cutoff period). We used global normalization to scale the mean of each scan to a common value in order to correct for whole brain differences over time at the first-level analysis for all individuals.

Data from each participant was entered into a general linear model using an event-related analysis procedure (Josephs \& Henson, 1999). Word pairs were treated as individual events for analysis and modeled using a canonical hemodynamic response function (HRF). Parameter estimates from contrasts of the canonical HRF in single participant models were entered into random-effects analysis across all participants to determine whether activation during a contrast was significant.

We first looked for activation when processing trained and transfer characters, relative to their perceptual baseline, separately for early and late sessions. Threshold was set at $p<0.05$ FWE-corrected with a cluster size of 10 or greater. Then, correlations between neural changes as a result of learning and behavioral performance were examined. Activation clusters were first identified at wholebrain level when threshold was set to $p<0.001$ uncorrected with a cluster size of 10 or greater. We further confirmed that these effects reached significance $(p<0.05)$ corrected for multiple comparisons, where a small volume correction (SVC, sphere of radius $10 \mathrm{~mm}$ ) was performed (Worsley et al., 1996), centered on the coordinates of each activation peak identified previously. For each activation cluster, Pearson correlation analysis were performed to estimate the correlation of the neural changes and behavioral performance, reported as $r$ value (correlation coefficient). Finally, in order to further investigate differences between the neural responses to trained versus transfer characters, we performed the regionof-interest (ROI) analysis for regions on activated regions in trained characters. By using the SPM Marsbar toolbox (Brett, Anton, Valabregue, \& Poline, 2002), ROI regions were constructed and the mean estimate of the percent signal change for each condition was calculated. We submitted these data for each region-ofinterest to a 2 novelty (trained, transfer) $\times 2$ session (early, late) $\times 2$ type (SI, $\mathrm{SU})$ analysis of variance (ANOVA) analysis.

\section{Results}

\subsection{Behavioral results}

Fig. 3 shows the behavioral performance (only accuracy) on quizzes and in the fMRI sessions. Because each subject started the training with different lists due to our counterbalancing, lists in each session were different among subjects. Therefore, the quiz scores were collapsed into three stages, in which all materials were learned once (stage 1), reviewed once (stage 2) and twice (stage 3), respectively. Performance on quizzes significantly increased in accuracy $[F(2,22)=12.686, p<0.001)]$ and decreased in reaction time (RT) $[F(2,22)=13.75, p<0.01]$ over time. Performance on SI characters demonstrated significantly higher accuracy $[F(1,11)=6.114, p<0.05]$ and faster RT $[F(1,11)=9.322, p<0.05]$ than SU characters. Responses 

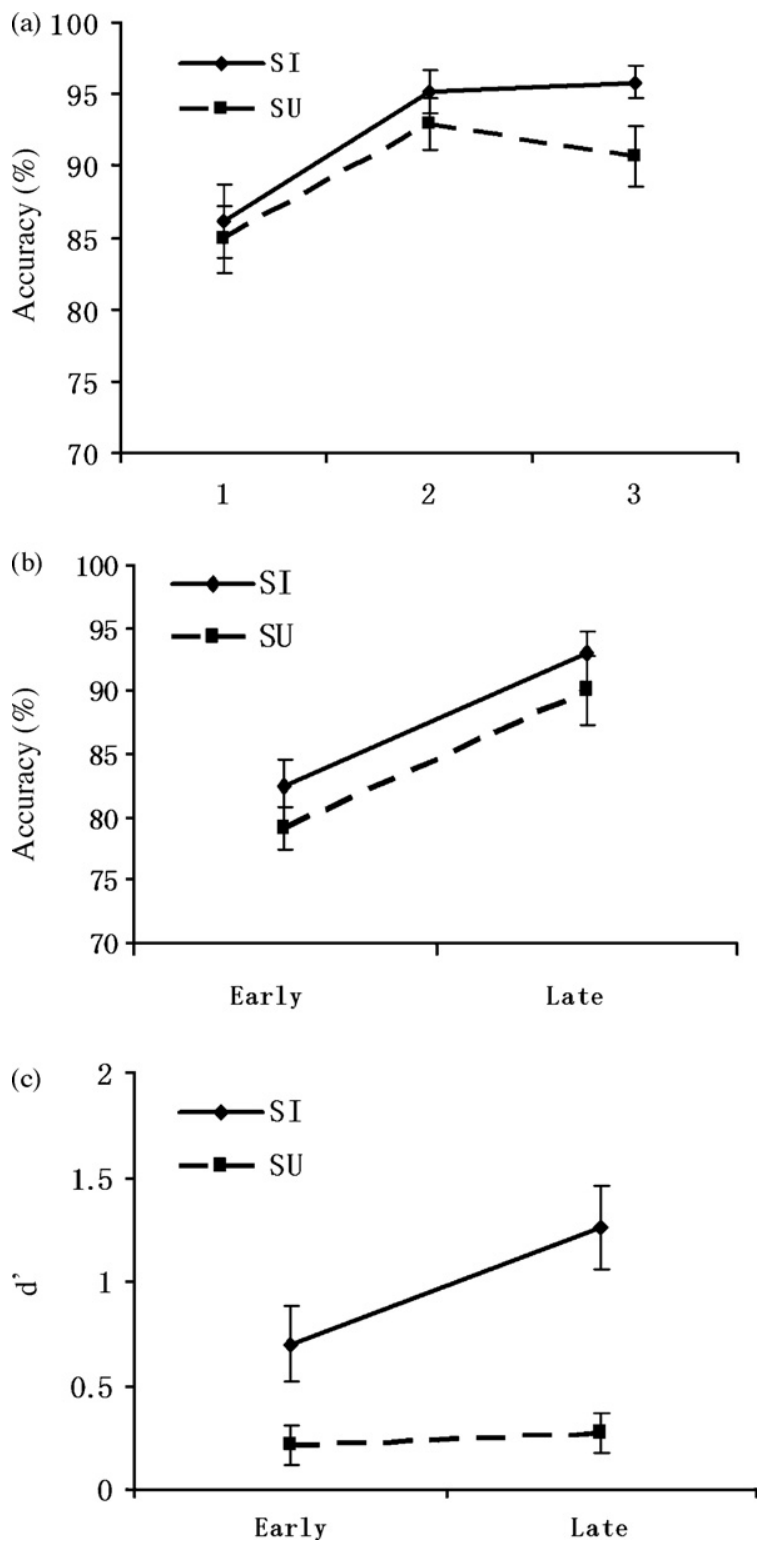

Fig. 3. Behavioral results. (a) Mean accuracy (bars indicate 1 standard error) for the quizzes for SI and SU character types: (1) collapsed data from all quizzes in the learning phase, (2) collapsed data from the first two quizzes in review phase, and (3) collapsed data from the third and fourth quizzes in review phase. (b) Mean accuracy (bars indicate 1 standard error) for trained characters in the early and late fMRI session. (c) Mean $d^{\prime}$ (bars indicate 1 standard error) for transfer characters in the early and late fMRI session. SI, semantic-implied; SU, semantic-unrelated.

to trained characters in the scanning sessions showed significantly higher accuracy $[F(1,11)=140.126, p<0.001]$ and faster RT $[F(1,11)=68.282, p<0.001]$ late versus early in learning. Accuracy for the SI type was significantly higher than that for the SU type $[F(1,11)=8.34, p<0.05]$ (Fig. 3b). There were no interactions between time and character type for the quizzes or the scanning sessions. For responses to transfer characters, the raw scores showed no reliable learning effect for either accuracy or reaction time. This lack of difference might be due to a stricter criterion while judging unlearned characters, which biased participants to reject the answer, so a sensitivity index $\left(d^{\prime}\right)$ was calculated to adjust for response bias. The value for $d^{\prime}$ is the $Z$ value of the hit-rate minus that of the false-alarmrate. This value indicates how sensitive an individual is to the experimental manipulation. As shown in Fig. 3c, an interaction between early/late learning and character type was found $[F(1,10)=10.391, p<0.01]$. SI type showed significantly higher $d^{\prime}$ late compared to early in learning $[F(1,10)=23.24, p<0.001]$, whereas $d^{\prime}$ for SU type did not differ over time [ $\left.p>0.8\right]$.

\subsection{Brain activations}

\subsubsection{Neural responses to trained characters}

There was no significant difference between SI and SU type characters on brain activation over time for trained characters, suggesting a similar learning/response strategy for both character types, so we collapsed across SI and SU type for imaging data analysis. Fig. 4a and Table 1 shows significantly activated areas for Chinese characters relative to the perceptual baseline early and late in learning. Overlapping activations were found in the left fusiform gyrus and middle occipital gyrus. For the late session, the activated regions extended to the left parahippocampus. In order to examine the learning effect, we correlated behavioral improvement with the activation changes from early to late learning, by entering the difference score in accuracy (late-early) as a covariate of interest in the contrast of greater activation late versus early in learning. Fig. $4 \mathrm{~b}$ and Table 1 presents both significant positive and negative correlations. Greater increases in accuracy were associated with stronger activation in the left superior parietal lobule/precuneus (BA7) $(r=0.89, p<0.001)$. However, greater increases in accuracy were associated with weaker activation in the left inferior frontal gyrus (BA47) $(r=-0.91, p<0.001)$.

\subsubsection{Neural responses to transfer characters}

According to the behavioral results on the transfer characters, SU and SI types showed significant differences, especially in the later session, indicating different learning/response strategies for each character type. In the early scan, there was no activation difference between characters and the perceptual baseline. In the late scan, both SI and SU showed greater activation in the left inferior frontal gyrus and fusiform gyrus (see Fig. 5a and Table 2). SU also showed activation in bilateral superior parietal cortex and the left medial frontal gyrus.

In order to determine whether brain activation during learning could predict maintenance of lexical knowledge, we examined the correlation between knowledge of radicals on a delayed recall test given 3-6 months after training and activation changes from early to late training, using behavioral performance as a covariate of interest in the contrast of late versus early in learning. We did this analysis only for the SI characters because processing these characters required the generalization of knowledge extracted from the trained semantic radicals to the transfer characters. As shown in Fig. 5b and Table 2, higher recall of semantic radicals in the delayed test was associated with greater activation in left precuneus (BA7) $(r=0.85$, $p<0.001)$. Other regions included bilateral middle frontal gyrus, right posterior cingulate and right middle temporal gyrus. 
a) Early Scan: Character $>$ Baseline
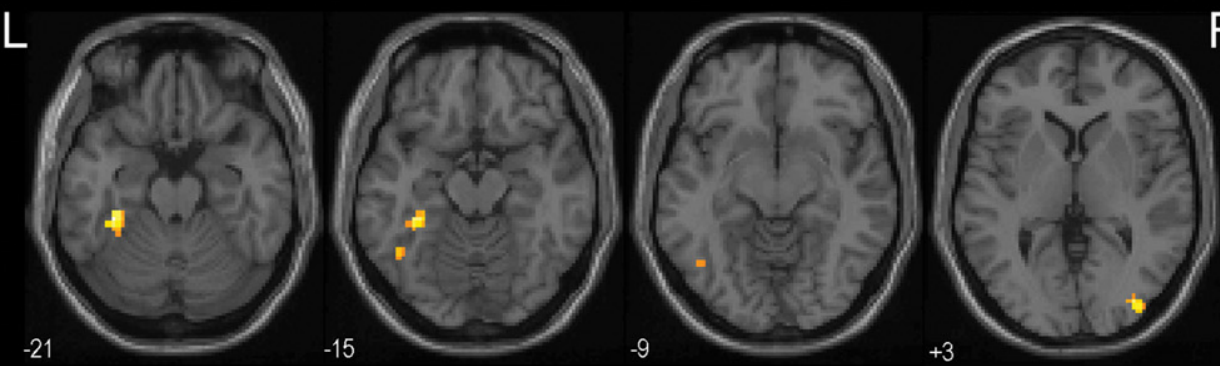

$\mathrm{R}$

15

10

5

0

\section{Late Scan: Character>Baseline}
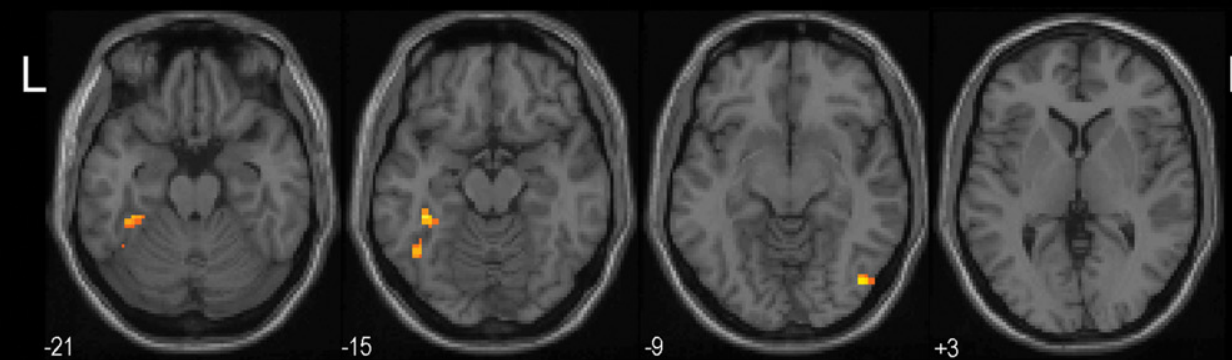

$\mathbf{R}$

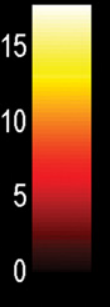

b)

Positive Correlation

Negative Correlation
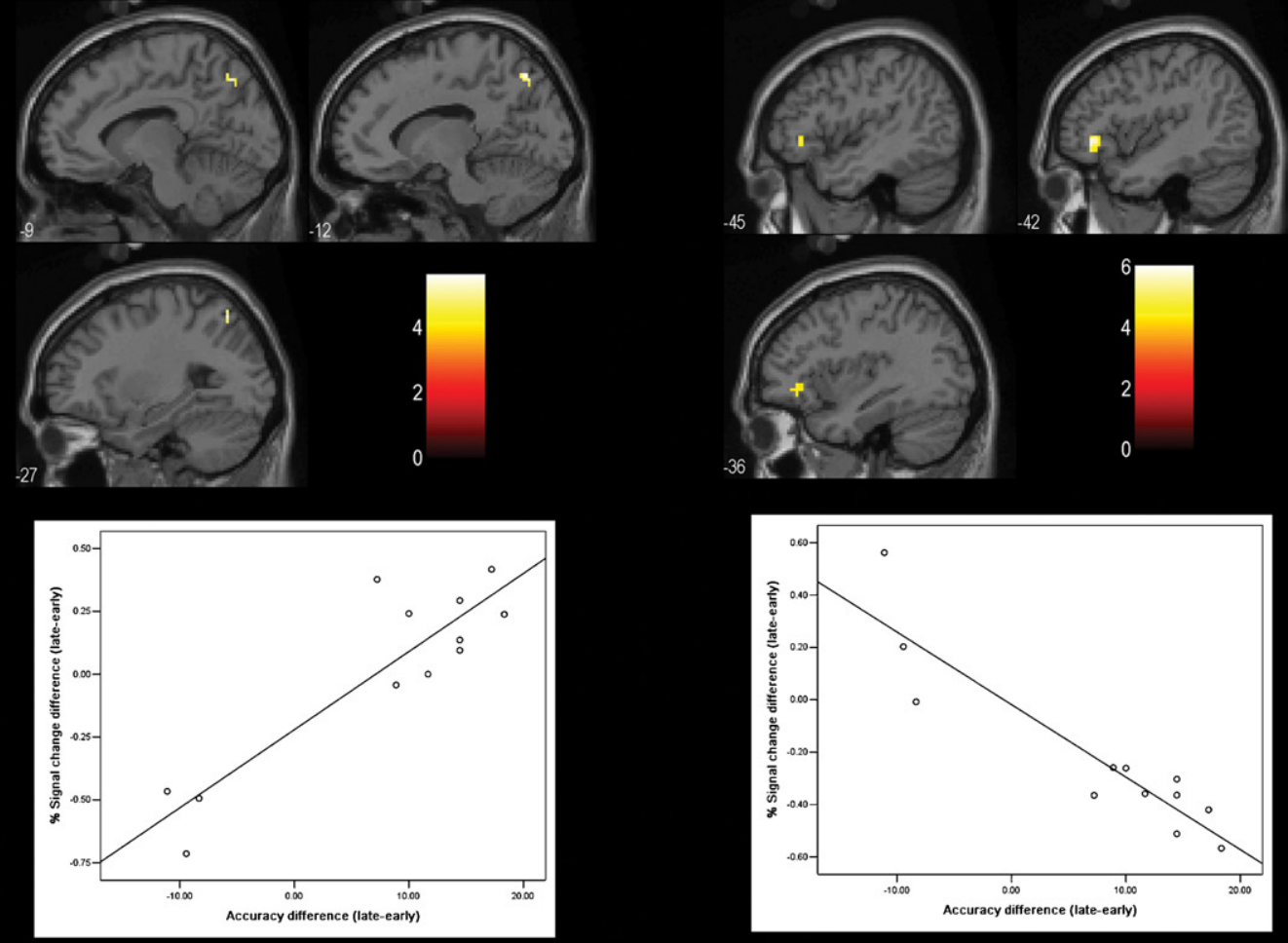

Fig. 4. (a) Brain activation maps for the semantic matching task (collapsed across SI and SU types) relative to a perceptual baseline for the trained characters in the early and late scan sessions. (b) Brain regions that showed greater activation for trained characters from early to late in learning that were positively (superior parietal lobule) and negatively (inferior frontal gyrus) correlated with greater accuracy improvement from early to late in learning. 
Table 1

Significant activations for trained characters and correlations between behavioral improvement and activation changes

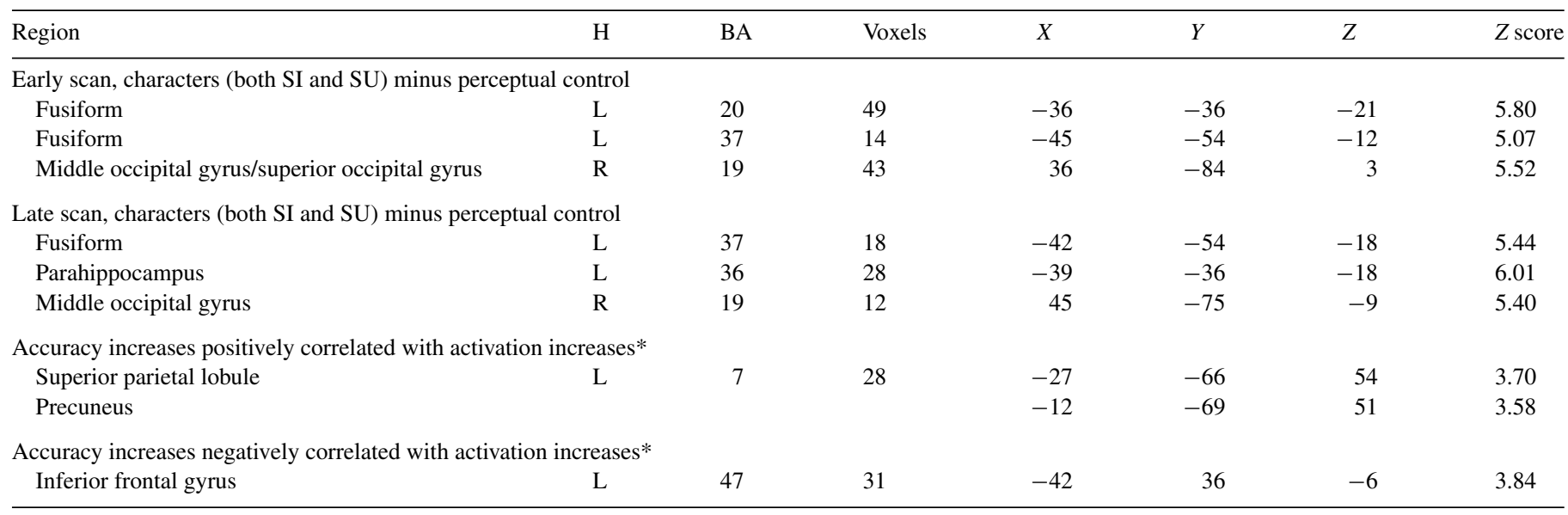

$\mathrm{H}=$ left $(\mathrm{L})$ or right $(\mathrm{R})$ hemisphere; BA = Brodmann's area of activation; voxels = number of voxels in cluster, only cluster 10 or greater are presented. $* p<0.05$ corrected after small-volume correction.

\subsubsection{Region-of-interest analyses}

In order to see how one generalizes trained knowledge to transfer items, we formed three regions of interest (ROIs) based on the peak of activation in clusters for the trained characters and applied those to the transfer characters. By doing the ROI analysis in this way, rather than selecting ROIs based on significance in the whole-brain analysis for transfer characters, we also avoided possible biases for inference (Poldrack, 2007). The three ROIs are: (1) the left fusiform gyrus ( -44 $-54-14$ ) based on the main effect of characters minus perceptual control, (2) the left superior parietal lobule (-27 -66 54) based on the positive correlation with accuracy, and (3) the left inferior frontal gyrus $(-4236-6)$ based on the negative correlation with accuracy. Radius of the ROIs was $3 \mathrm{~mm}$ in order to avoid extending into another anatomically defined area. For each subject, we calculated the percentage signal changes for all experimental conditions, in which the baseline tasks were included in the contrast in order to control the between-session variations. In order to examine whether learning effects differed for trained versus transfer characters, we performed a 2 novelty (trained, transfer) $\times 2$ session (early, late) $\times 2$ type $(\mathrm{SI}$, SU) ANOVA analysis separately for each region. As shown in Fig. 6, there were several significant interactions. In the fusiform region (Fig. 6a), a novelty by session interaction was found $[F(1,11)=6.016, p<0.05]$. Post hoc analysis showed that there was no learning effect for the trained characters, whereas there was an increase in activation from early to late for the transfer characters $[F(1,11)=7.426, p<0.05]$, driven primarily by the SU type. In superior parietal lobule region (Fig. 6b), a main effect of type $[F(1,11)=9.151, p<0.05]$, and a novelty by session interaction were found $[F(1,11)=4.415, p=0.05]$. Post hoc analysis showed that activation reduced from early to

Table 2

Significant activations for transfer characters and correlations between scores of recall test and activation increases

\begin{tabular}{|c|c|c|c|c|c|c|c|}
\hline Region & $\mathrm{H}$ & BA & Voxels & $X$ & $Y$ & $Z$ & $Z$ score \\
\hline Inferior frontal gyrus & $\mathrm{L}$ & $45 / 46$ & 56 & -51 & 33 & 6 & 5.99 \\
\hline \multicolumn{8}{|c|}{ Late scan: semantic-unrelated minus perceptual control in late session } \\
\hline Inferior frontal gyrus & $\mathrm{L}$ & 47 & 53 & -51 & 36 & 0 & 6.48 \\
\hline Medial frontal gyrus & $\mathrm{L}$ & 8 & 15 & -12 & 36 & 48 & 5.19 \\
\hline Middle temporal gyrus & $\mathrm{L}$ & $21 / 37$ & 30 & -57 & -51 & -9 & 5.13 \\
\hline Fusiform & & & & -45 & -54 & -15 & 5.12 \\
\hline \multicolumn{8}{|c|}{ Activation increases correlated with recognition of radicals* } \\
\hline Precuneus & $\mathrm{L}$ & 7 & 13 & -36 & -66 & 36 & 3.49 \\
\hline Posterior cingulate & $\mathrm{R}$ & 23 & 14 & 15 & -54 & 9 & 3.93 \\
\hline
\end{tabular}

$\mathrm{H}=$ left $(\mathrm{L})$ or right $(\mathrm{R})$ hemisphere; BA = Brodmann's area of activation; voxels = number of voxels in cluster, only cluster 10 or greater are presented. * $p<0.05$ corrected after small-volume correction. 


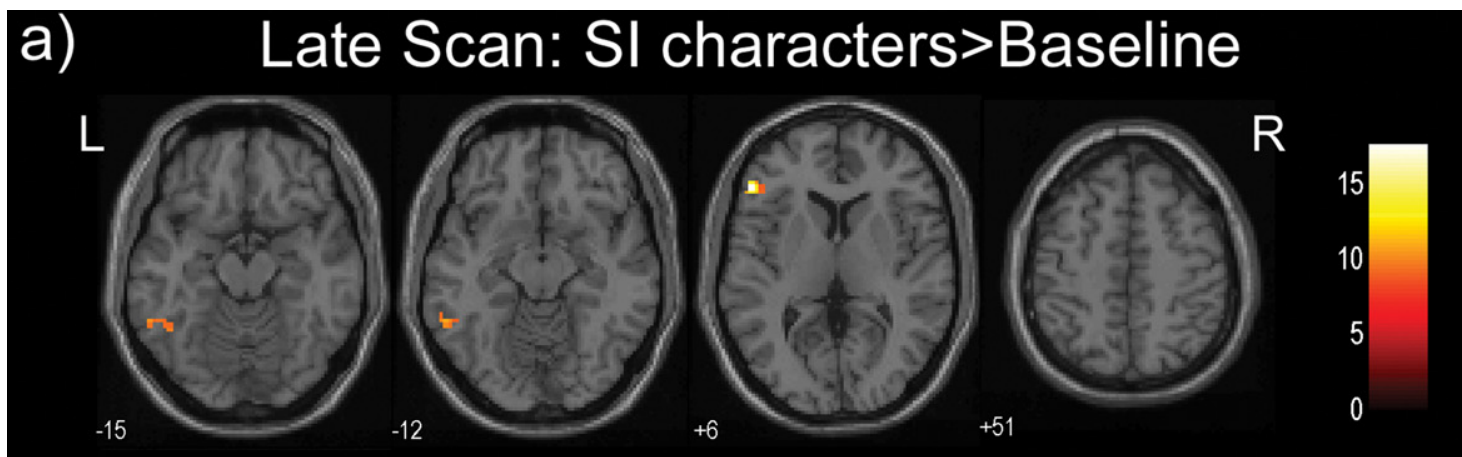

\section{Late Scan: SU characters>Baseline}
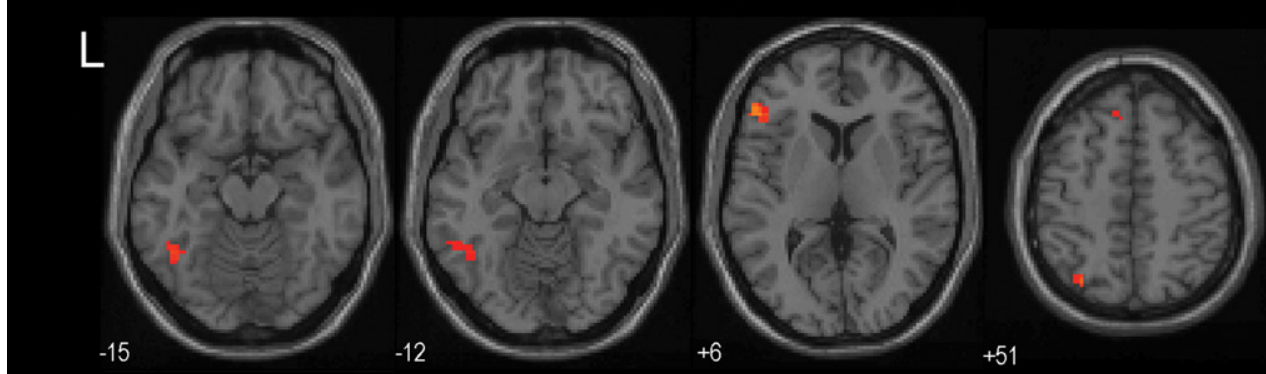

$\mathrm{R}$
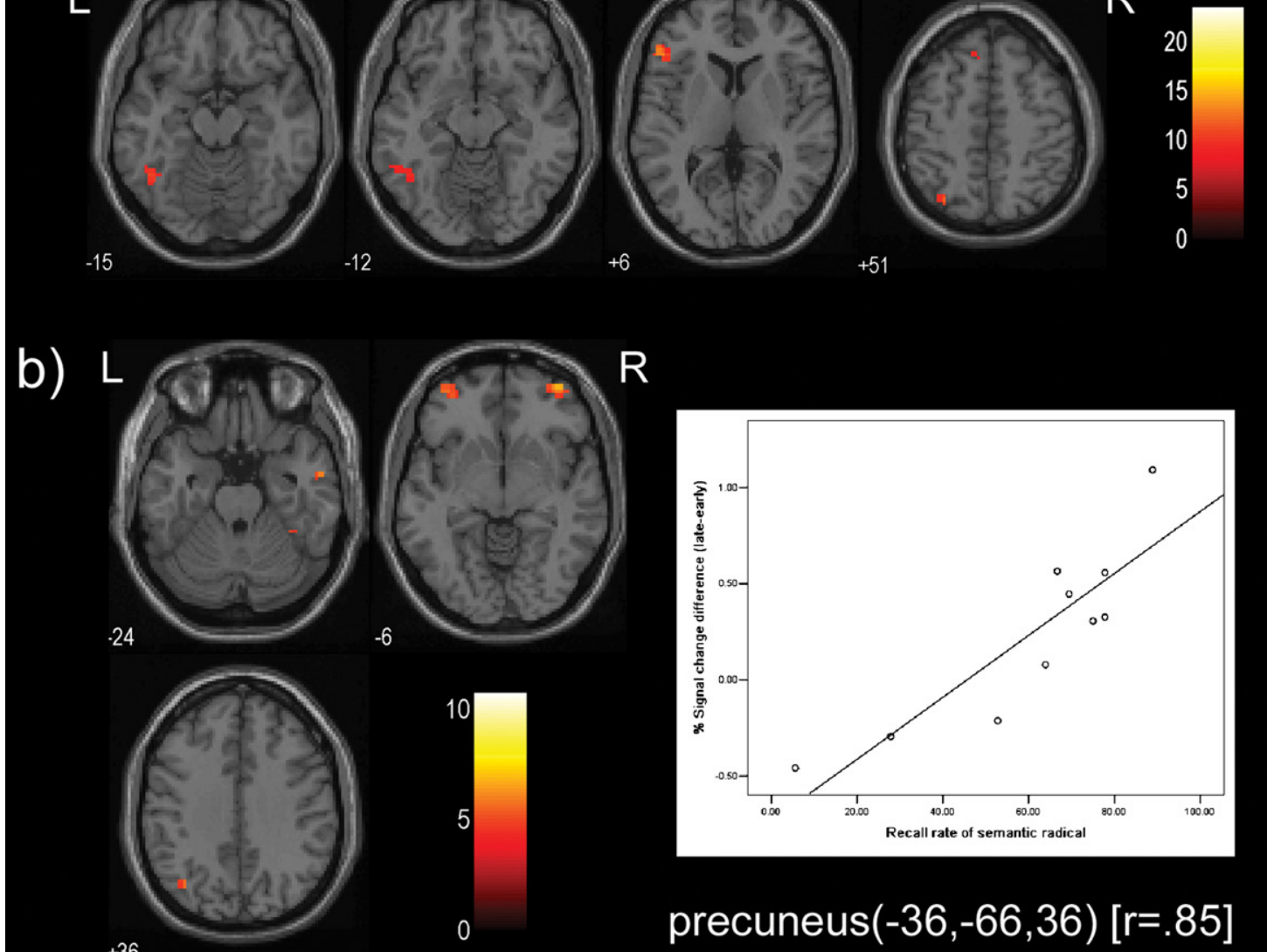

Fig. 5. (a) Brain activation maps for the semantic matching task relative to a perceptual baseline for the transfer characters for SI and SU types in the late scan session. (b) Brain regions that showed greater activation for transfer characters from early to late in learning that were positively correlated with higher recognition rate of radicals in a delayed test given 3-6 months after training. Please note that only 10 of the 12 subjects were available for the delayed testing.

late for the trained characters $[F(1,11)=8.85, p<0.05]$ driven primarily by the SI type, whereas there was no learning effect for the transfer characters. In inferior frontal gyrus region (Fig. 6c), a main effect of novelty $[F(1,11)=10.35, p<0.01]$ and a novelty by session interaction were found $[F(1,11)=7.084$, $p<0.05]$. Post hoc analysis showed that activation reduced from early to late for trained characters $[F(1,11)=5.74, p<0.05]$ driven primarily by the SU type, and activation increased from early to late for transfer characters $[F(1,11)=38.82$, $p<0.001]$.

\section{Discussion}

Neural changes resulting from English speakers learning the meaning of Chinese characters were examined in a crossmodality matching task using fMRI. The present study differed from previous lexical learning studies because it allowed participants to implicitly learn sub-lexical semantic knowledge during training and transfer of semantic knowledge was measured by examining the processing of novel characters that shared a semantic radical with trained characters. Behavioral findings 

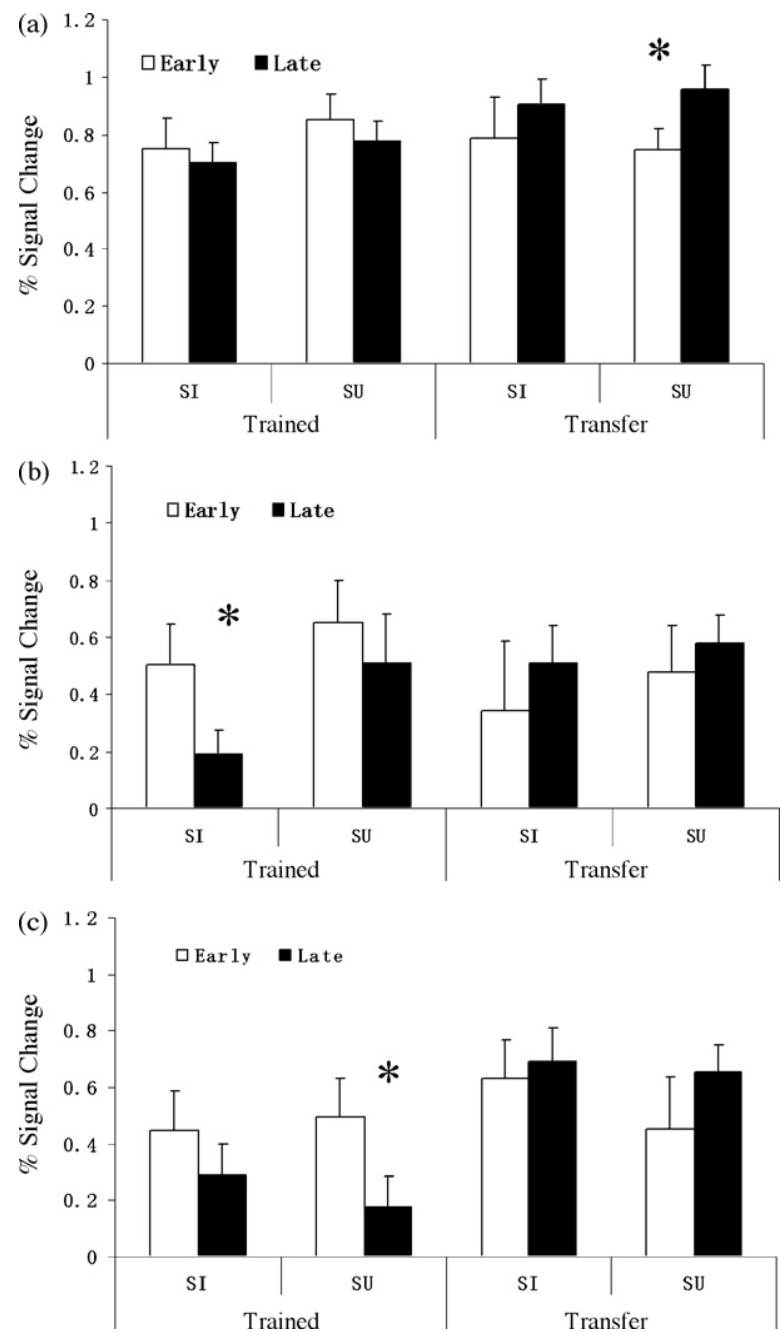

Fig. 6. Percentage signal change for SI and SU types on both trained and transfer characters in three ROIs: (a) fusiform gyrus, (b) superior parietal lobule, and (c) inferior frontal gyrus. Asterisks indicates a significant difference from early to late in training $(t$-test $p<0.05)$.

suggested that the acquisition of underlying semantic knowledge facilitated the recognition of both trained and transfer characters. For imaging results, there were selective activation patterns in three left lateral cortical areas, including the fusiform gyrus, the superior parietal lobule and the inferior frontal gyrus, indicating their distinct roles in lexical learning and transfer.

\subsection{Learning effect on trained characters}

Several lines of research suggest that the left fusiform gyrus is important for processing orthographic information (Nobre, Allison, \& McCarthy, 1994; Petersen, Fox, Snyder, \& Raichle, 1990) and developmental imaging studies have shown that activation of this region increases with age and correlates with reading skill (Shaywitz et al., 2002). In current study, the left fusiform gyrus showed robust activation for the lexical condition (characters) relative to a perceptual baseline (meaningless visual symbols), supporting its key role in visual processing of orthographic information irrespective of the written system. Evidence from lexical learning studies have also shown learning effects in the fusiform gyrus (Breitenstein et al., 2005; Hashimoto \& Sakai, 2004; Poldrack et al., 1998; Sandak et al., 2004; Xue et al., 2006). For example, an artificial language learning study (Xue et al., 2006) reported that activation in the midfusiform region decreased after a short period of visual word form learning, but increased in subsequent phonological and semantic learning. It has also been suggested that, as proficiency improves, processing of a new language might be associated with a shift from rule-based analysis in a dorsal parietal pathway to pattern-based recognition in a ventral temporal pathway (Hashimoto \& Sakai, 2004; Poldrack et al., 1998). In our study, however, fusiform activation was statistically comparable between early and late in learning, indicating that the learning of Chinese characters may still have been at an early stage of learning.

Although the ROI analyses reveal an overall decrease of activation in SPL with training, correlation analyses show that learning related activation in SPL was also modulated by behavior. Greater improvement on subjects' performance from early to late in learning was correlated with greater activation in the left superior parietal lobule. Superior parietal lobule activation has been consistently found in most imaging studies of Chinese characters across different tasks and conditions, including homophone judgment (Kuo et al., 2004; Tan, Feng et al., 2001), rhyming judgment (Booth et al., 2006), processing words with short presentation duration (Fu, Chen, Smith, Iversen, \& Matthews, 2002; Peng et al., 2003), irregular character processing (Lee et al., 2004; Tan, Liu et al., 2001), processing of words with vague meanings (Tan et al., 2000), and processing low frequency words (Kuo et al., 2003). Lee et al. (2004) found a frequency by consistency interaction for Chinese character processing in the temporo-parietal region, suggesting the importance of this region in rule-based analysis at the sub-lexical level. Additionally, visual attention studies have found superior parietal lobule/lateral precuneus activation in tasks that involve shifting of attention (Booth et al., 2004; Cohen et al., 2002) and spatial working memory (Buckner, Raichle, Miezin, \& Petersen, 1996; Shallice et al., 1994). The superior parietal lobule may be associated with a top-down allocation of attention and conjunction functioning by binding separately presented local features (Corbetta et al., 1995; Kanwisher \& Wojciulik, 2000; Kuo et al., 2003, 2004). In a post-test questionnaire, most subjects (11 out of 12) in our study reported that they noticed the meanings of similar characters belonged to a specific category and most linked the visual features and meanings of a single character to similar unlearned transfer characters (9 out of 11). Therefore, the acquisition of Chinese characters in English speakers may have involved an increasing demand on visual-spatial processing of visual features within characters (i.e. semantic radical), resulting in larger learning effect in the superior parietal lobule for those who showed greater improvement from early to late in learning. However, overall activation in the superior parietal lobule, especially for the SI type characters, decreased from early to late in learning suggesting that this region became more efficient in visual-spatial processing. Taken together, these studies suggest that the superior parietal lobule may play a crucial role in the sub-lexical (i.e. radical) processing of the visual features of Chinese characters. 
In the present study, greater behavioral improvement from early to late in learning was associated with greater decreases in activation in the inferior frontal gyrus from early to late in learning. Evidence from priming studies has shown that repetition of stimuli results in decreased neural activity in the inferior frontal region during semantic processing (Buckner, Koutstaal, Schacter, \& Rosen, 2000; Wagner, Koutstaal, Maril, Schacter, \& Buckner, 2000). Similarly, studies of lexical learning have reported reduced activation in left inferior frontal gyrus when stimuli are repeated (Poldrack et al., 2001; Sandak et al., 2004). Bilingual studies also have shown that higher proficiency in semantic processing is associated with less activation of the inferior frontal gyrus region (Chee, Hon, Lee, \& Soon, 2001; Perani et al., 2003). Our results are consistent with these studies and suggest the reduced inferior frontal gyrus may be related to more efficient processing of semantic information over learning. However, the training related decrease, across the whole group, from early to late in learning was more pronounced for the SU type characters, which also had lower accuracy. The SI type characters shared semantic radicals that gave a clue to meaning, whereas the SU characters shared phonological radicals so they were just visually similar. It is possible that the smaller reduction for SI type characters was because subjects were continuing to use the radicals to access meaning, whereas the SU radicals were just visually similar and therefore the greater reduction could represent perceptual repetition effect.

\subsection{Learning effect on transfer characters}

In the present study, lexical learning involved the implicit learning of semantic radicals and the transfer of learned knowledge facilitated behavioral responses to novel characters. SU type characters in which transfer of semantic information about the radical was not possible, produced more activation in the superior parietal lobule as compared to SI type characters in which the transfer of semantic information was possible. It may be that SU character processing demands more visual-spatial attention to segment radicals (Booth et al., 2006; Chen, Fu, Iversen, Smith, \& Matthews, 2002; Fu et al., 2002). We also found that better performance in a delayed test (3-6 months after training) of radical recall was associated with a greater activation increase from early to late in learning for transfer characters in the left lateral precuneus. Better performance on radical recall presumably indicates the participants' knowledge of the underlying lexical rule that associates radicals with semantic category information. Even without explicit instruction, many of our participants acquired the relationship between radicals and whole characters after training and attempted to transfer the acquired radical knowledge to the novel characters that shared the same semantic radical. As discussed above, the dorsal aspect of parietal region, including the superior parietal lobule and the lateral aspect of precuneus, appears to be involved in the visual-spatial processing of sub-lexical components of Chinese characters, therefore it is possible that reliance on this neuro-cognitive network resulted in better learning over the long term.

Convergent evidence from neuroimaging studies on the processing of written alphabetic words has suggested that the left inferior parietal cortex (BA40) mediates the integration between lexical representations (Booth et al., 2002a; Chou, Booth, Bitan et al., 2006; Chou, Booth, Burman et al., 2006). Learning-related changes are also found in this area (Breitenstein et al., 2004; Lee et al., 2003; Poldrack et al., 1998). Evidence from remediation studies (Eden et al., 2004; Kujala et al., 2001; Temple et al., 2003) also report that, after a phonic/phonological-based training, dyslexics show increased activation in the left inferior parietal lobule, which usually shows less activation in dyslexics compared to controls. A recent meta-analysis, however, suggests that the left posterior sites of temporo-parietal regions are important for alphabetic languages, but not for Chinese characters (Tan et al., 2005). The dorsal aspect of parietal region (BA7) seems more crucial for the visual-spatial processing of Chinese characters. The present results also support this notion by showing learning and transfer effects for Chinese character processing in the superior parietal lobule.

Although there were no correlations between behavioral performance and activation in the fusiform gyrus, there were increases in activation from early to late in learning for the transfer characters. This increase was primarily driven by the SU type characters which did not show any behavioral improvement over learning. SU type characters are more difficult than SI characters because the former cannot benefit from the transfer of knowledge about the semantic radical. Other training studies have shown greater activation in the fusiform region for characters that are less familiar or more difficult (Xue et al., 2006). The mechanism for the difficulty modulation in this region is unclear, as the fusiform region could be involved in orthographic processing (Baker et al., 2007; Cohen \& Dehaene, 2004) or integration functions such as polymodal interactions to visual, auditory and tactile inputs (Price \& Devlin, 2003). In our study, it is most likely that greater activation in the fusiform gyrus over learning may be related to transferring orthographic knowledge to SU characters. The posterior part of middle temporal gyrus (BA21) also showed greater activation for transfer characters in the late scan as compared to the early scan, and no learning effect was found for trained characters in this region. This region is thought to be involved in semantic processing (Castillo et al., 2000; Gold \& Buckner, 2002; also see Price, 2000 for a review) across different scripts (Nakamura, Dehaene, Jobert, Bihan, \& Kouider, 2005) and independent of input modality (Booth et al., 2002b). Therefore, the learning-related increases in activation of the middle temporal gyrus may reflect the more effective access of semantic information when processing transfer characters.

Activation in the left inferior frontal gyrus has been shown for variety of semantic tasks in English (Demb et al., 1995; Thompson-Schill, D'esposito, Aguirre, \& Farah, 1997; Wagner, Pare-Blagoev, Clark, \& Poldrack, 2001). Some have argued that the inferior frontal gyrus is a semantic working memory system or semantic executive system that is involved in the controlled retrieval, selection, and evaluation of semantic representations (Gabrieli, Poldrack, \& Desmond, 1998; Gold \& Buckner, 2002; Poldrack et al., 1999; Wagner et al., 2001). As in English, left inferior frontal gyrus has also been found to be associated with control processes in the semantic processing of Chinese 
characters (Zhang et al., 2004). In the present study, we found that transfer characters produced greater activation than trained characters both early and late in learning and that there was an increase from early to late in learning for the transfer characters in the ventral inferior frontal gyrus (BA47). These effects suggest that participants may be more effectively engaging executive processes in the inferior frontal gyrus when processing transfer characters, especially late in learning. In fact, the behavioral measures showed that participants were performing near chance levels early in learning and had a bias of responding no, whereas later in learning participants were scoring reliably above chance. We did not find activation in the dorsal aspect of the inferior frontal gyrus (BA9) in any of the comparisons in this paper for either the trained or transfer characters. The dorsal part of inferior frontal gyrus is thought to play a key role in addressed phonology of Chinese characters (Tan et al., 2005). However, the pronunciations of Chinese characters were not provided in this study and no subjects reported they concentrated on the pronunciation of the English words, so this may explain the lack of recruitment of the dorsal aspect of the inferior frontal gyrus.

\section{Conclusion}

In this study, we took advantage of the unique characteristic of Chinese phonograms to examine both the item-specific learning effects on trained characters and the generalization of semantic knowledge to transfer characters on brain activation. Our findings suggest that the left fusiform gyrus is involved in the orthographic processing of characters and that the inferior frontal gyrus is associated with retrieval and manipulation of semantic representations. More importantly, the present study, for the first time, demonstrates the role of the left superior parietal cortex in learning the visual-spatial aspects of Chinese characters and also generalization of orthographic-semantic knowledge to novel characters.

\section{Acknowledgements}

This work was supported by grants from the Chinese Natural Science Foundation (Project 30270462 and Project 30400133). This work was also supported by grants from the National Institute of Child Health and Human Development (HD042049), and by the National Institute of Deafness and Other Communication Disorders (DC06149) to JRB. This study was conducted at Northwestern University, Evanston, IL 60208, USA.

\section{References}

Baayen, R. H., Piepenbrock, R., \& Gulikers, L. (1995). The CELEX lexical database (version release 2) [CD-ROM]. Philadelphia: Linguistic Data Consortium, University of Pennsylvania.

Baker, C. I., Liu, J., Wald, L. L., Kwong, K. K., Benner, T., \& Kanwisher, N. (2007). Visual word processing and experiential origins of functional selectivity in human extrastriate cortex. Proceedings of the National Academy of Sciences of the United States of American, 104, 9087-9092.

Bolger, D. J., Perfetti, C. A., \& Schneider, W. (2005). Cross-cultural effect on the brain revisited: Universal structures plus writing system variation. Human Brain Mapping, 25, 92-104.
Booth, J. R., Burman, D. D., Meyer, J. R., Gitelman, D. R., Parrish, T. B., \& Mesulam, M. M. (2002a). Functional anatomy of intra- and cross-modal lexical tasks. Neuroimage, 16, 7-22.

Booth, J. R., Burman, D. D., Meyer, J. R., Gitelman, D. R., Parrish, T. B., \& Mesulam, M. M. (2002b). Modality independence of word comprehension. Human Brain Mapping, 16, 251-261.

Booth, J. R., Burman, D. D., Meyer, J. R., Gitelman, D. R., Parrish, T. B., \& Mesulam, M. M. (2004). Development of brain mechanisms for processing orthographic and phonologic representations. Journal of Cognitive Neuroscience, 16, 1234-1249.

Booth, J. R., Lu, D., Burman, D. D., Chou, T. L., Jin, Z., Peng, D. L., et al. (2006). Specialization of phonological and semantic processing in Chinese word reading. Brain Research, 1071, 197-207.

Breitenstein, C., Jansen, A., Deppe, M., Foerster, A. F., Sommer, J., Wolbers, T., et al. (2005). Hippocampus activity differentiates good from poor learners of a novel lexicon. Neuroimage, 25, 958-968.

Brett, M., Anton, J. -L., Valabregue, R., \& Poline, J. -B. (2002). Region of interest analysis using an SPM toolbox [abstract]. Presented at the 8th International Conference on Functional Mapping of the Human Brain, June 2-6, 2002, Sendai, Japan. Available on CD-ROM in NeuroImage, Vol. 16, No. 2.

Buchel, C., Coull, J. T., \& Friston, K. J. (1999). The predictive value of changes in effective connectivity for human learning. Science, 283, 15381541 .

Buckner, R. L., Raichle, M. E., Miezin, F. M., \& Petersen, S. E. (1996). Functional anatomic studies of memory retrieval for auditory words and visual pictures. Journal of Neuroscience, 16, 6219-6235.

Buckner, R. L., Koutstaal, W., Schacter, D. L., \& Rosen, B. R. (2000). Functional MRI evidence for a role of frontal and inferior temporal cortex in amodal components of priming. Brain, 123, 620-640.

Callan, D. E., Tajima, K., Callan, A. M., Kubo, R., Masaki, S., \& Yamada, R. A. (2003). Learning-induced neural plasticity associated with improved identification performance after training of a difficult second-language phonetic contrast. Neuroimage, 19, 113-124.

Castillo, E. M., Simos, P. G., Davis, R. N., Breier, J., Fitzgerald, M. E., \& Papanicolaou, A. C. (2000). Levels of word processing and incidental memory: Dissociable mechanisms in the temporal lobe. Neuroreport, 12, 35613566.

Chee, M. W. L., Tan, E. W., \& Thiel, T. (1999). Mandarin and English single word processing studied with functional magnetic resonance imaging. Journal of Neuroscience, 19, 3050-3056.

Chee, M. W. L., Weekes, B., Lee, K. M., Soon, C. S., Schrieber, A., Hoon, J. J., et al. (2000). Overlap and dissociation of semantic processing of Chinese characters English words and pictures: Evidence from fMRI. Neuroimage, 12, 392-403.

Chee, M. W. L., Hon, N., Lee, H. L., \& Soon, C. S. (2001). Relative language proficiency modulates BOLD signal change when bilinguals perform semantic judgments. Neuroimage, 13, 1155-1163.

Chen, Y. P., Fu, S., Iversen, S. D., Smith, S. M., \& Matthews, P. M. (2002). Testing for dual brain processing route in readings: A direct contrast of Chinese character and Pinyin reading using fMRI. Journal of Cognitive Neuroscience, 14, 1088-1098.

Chou, T. L., Booth, J. R., Bitan, T., Burman, D. D., Bigio, J. D., Cone, N. E., et al. (2006). Developmental and skill effects on the neural correlates of semantic processing to visually presented words. Human Brain Mapping, 27, 915-924.

Chou, T. L., Booth, J. R., Burman, D. D., Bitan, T., Bigio, J. D., Dong, L., et al. (2006). Developmental differences in the neural correlates of semantic judgments to auditorily presented word pairs. Neuroimage, 29, 11411149.

Clark, D., \& Wagner, A. D. (2003). Assembling and encoding word representations: fMRI subsequent memory effects implicate a role for phonological control. Neuropsychologia, 41, 304-317.

Cohen, L., \& Dehaene, S. (2004). Specialization within the ventral stream: The case for the visual word form area. Neuroimage, 22, 466-476.

Cohen, L., Lehericy, S., Chochon, F., Lemer, C., Rivaud, S., \& Dehaene, S. (2002). Language-specific tuning of visual cortex? Functional properties of the visual word form area. Brain, 125, 1054-1069. 
Corbetta, M., \& Shulman, G. L. (2002). Control of goal-directed and stimulus driven attention in the brain. Nature Review Neuroscience, 3, 201-215.

Demb, J. B., Desmond, J. E., Wagner, A. D., Vaidya, C. J., Glover, G. H., \& Gabrieli, J. D. E. (1995). Semantic encoding and retrieval in the left inferior prefrontal cortex: A functional MRI study of task difficulty and process specificity. Journal of Neuroscience, 15, 5870-5878.

Dolan, R. J., Fink, G. R., Rolls, E., Booth, M., Holmes, A., Frackowiak, R. S. J., et al. (1997). How the brain learns to see objects and faces in an impoverished context. Nature, 389, 596-599.

Eden, G. F., Jones, K. M., Cappell, K., Gareau, L., Wood, F. B., Zeffiro, T. A., et al. (2004). Clinical neural changes following study remediation in adult developmental dyslexia. Neuron, 44, 411-422.

Fu, S. M., Chen, Y. P., Smith, S., Iversen, S., \& Matthews, P. M. (2002). Effects of word form on brain processing of written Chinese. Neuroimage, 17, 1538-1548.

Gabrieli, J. D. E., Poldrack, R. A., \& Desmond, J. E. (1998). The role of left prefrontal cortex in language and memory. Proceedings of the National Academy of Science, 95, 906-913.

Gauthier, I., Tarr, M. J., Anderson, A. W., Skudlarski, P. S., \& Gore, J. C. (1999). Activation of the middle fusiform 'face area' increases with expertise in recognizing novel objects. Nature Neuroscience, 2, 568-573.

Gold, B. T., \& Buckner, R. L. (2002). Common prefrontal regions coactivate with dissociable posterior regions during controlled semantic and phonological tasks. Neuron, 35, 803-812.

Hashimoto, R., \& Sakai, K. L. (2004). Learning letters in adulthood: Direct visualization of cortical plasticity for forming a new link between orthography and phonology. Neuron, 42, 311-322.

Josephs, O., \& Henson, R. N. A. (1999). Event-related fMRI: Modelling, inference and optimisation. Philosophical Transactions of the Royal Society of London, 354, 1215-1228.

Kanwisher, N., \& Wojciulik, E. (2000). Visual attention: Insights from brain imaging. Nature Review Neuroscience, 1, 91-100.

Kujala, T., Karma, K., Ceponiene, R., Belitz, S., Turkkila, P., Tervaniemi, M., et al. (2001). Plastic neural changes and reading improvement caused by audiovisual training in reading-impaired children. Proceedings of the National Academy of Science, 98, 10509-10514.

Kuo, W. J., Yeh, T. C., Lee, C. Y., Wu, Y. T., Chou, C. C., Ho, L. T., et al. (2003). Frequency effects of Chinese character processing in the brain: An event-related fMRI study. Neuroimage, 18, 720-730.

Kuo, W. J., Yeh, T. C., Lee, J. R., Chen, L. F., Lee, P. L., Chen, S. S., et al. (2004). Orthographic and phonological processing of Chinese characters: An fMRI study. Neuroimage, 21, 1721-1731.

Lee, C. Y., Tsai, J. L., Kuo, W. J., Yeh, T. C., Wu, Y. T., Ho, L. T., et al. (2004). Neuronal correlates of consistency and frequency effects on Chinese character naming: An event-related fMRI study. Neuroimage, 23, 1235 1245.

Lee, H. S., Fujii, T., Okuda, J., Tsukiura, T., Umetsu, A., Suzuki, M., et al. (2003). Changes in brain activation patterns associated with learning of Korean words by Japanese: An fMRI study. Neuroimage, 20, 1-11.

Nakamura, K., Dehaene, S., Jobert, A., Bihan, D. L., \& Kouider, S. (2005). Subliminal convergence of Kanji and Kana words: Further evidence for functional parcellation of the posterior temporal cortex in visual word perception. Journal of Cognitive Neuroscience, 17, 954-968.

Nelson, D. L., McEvoy, C. L., \& Schreiber, T. A. (1998). The University of South Florida word association, rhyme, and word fragment norms http://w3.usf.edu/FreeAssociation/.

Nobre, A. C., Allison, T., \& McCarthy, G. (1994). Word recognition in the human inferior temporal lobe. Nature, 372, 260-263.

Peng, D. L., Xu, D., Jin, Z., Luo, Q., Ding, G. S., Perry, C., et al. (2003). Neural basis of the non-attentional processing of briefly presented words. Human Brain Mapping, 18, 215-221.

Perani, D., Abutalebi, J., Paulesu, E., Brambati, S., Scifo, P., Cappa, S. F., et al. (2003). The role of age of acquisition and language usage in early, highproficient bilinguals: An fMRI study during verbal fluency. Human Brain Mapping, 19, 170-182.

Petersen, S. E., Fox, P. T., Snyder, A. Z., \& Raichle, M. E. (1990). Activation of extrastriate and frontal cortical areas by visual words and word-like stimuli. Science, 249, 1041-1044.
Poldrack, R. A. (2007). Region of interest analysis for fMRI. Social Cognitive \& Affective Neuroscience, 2, 67-70.

Poldrack, R. A., Desmond, J. E., Glover, G. H., \& Gabrieli, J. D. E. (1998). The neural basis of visual skill learning: An fMRI study of mirror reading. Cerebral Cortex, 8, 1-10.

Poldrack, R. A., Wagner, A. D., Prull, M. W., Desmond, J. E., Glover, G. H., \& Gabrieli, J. D. E. (1999). Functional specialization for semantic and phonological processing in the left inferior prefrontal cortex. Neuroimage, 10, 15-35.

Price, C. (2000). The anatomy of language: Contributions from functional neuroimaging. Journal of Anatomy, 197, 335-359.

Price, C. J., \& Devlin, J. T. (2003). The myth of the visual word form area. Neuroimage, 19, 473-481.

Raboyeau, G., Marie, N., Balduyck, S., Gros, H., Demonet, J. F., \& Cardebat, D. (2004). Lexical learning of the English language: A PET study in healthy French subjects. Neuroimage, 22, 1808-1818.

Rosenberg, D. R., Sweeney, J. A., Gillen, J. S., Chang, S. Y., Varanelli, M. J., O'Hearn, K., et al. (1997). Magnetic resonance imaging of children without sedation: Preparation with simulation. Journal of the American Academy of Child and Adolescent Psychiatry, 36, 853-859.

Sandak, R., Mencl, W. E., Frost, S. J., Rueckl, J. G., Katz, L., Moore, D. L., et al. (2004). The neurobiology of adaptive learning in reading: A contrast of different training conditions. Cognitive Affective \& Behavioral Neuroscience, 4, 67-88.

Shallice, T., Fletcher, P. C., Frith, C. D., Grasby, P. K., Frackowiak, R. S., \& Dolan, R. J. (1994). Brain regions associated with acquisition and retrieval of verbal episodic memory. Nature, 368, 633-635.

Shaywitz, B. A., Shaywitz, S. E., Pugh, K. R., Mencl, W. E., Fulbright, R. K., Skudlarski, P., et al. (2002). Disruption of posterior brain systems for reading in children with developmental dyslexia. Biological Psychiatry, 52, 101-110.

Stein, M., Dierks, T., Brandeis, D., Wirth, M., Strik, W., \& Koenig, T. (2006). Plasticity in the adult language system: A longitudinal electrophysiological study on second language learning. Neuroimage, 33, 774-783.

Tan, L. H., Feng, C. M., Fox, P. T., \& Gao, J. H. (2001). An fMRI study with written Chinese. Neuroreport, 12, 83-88.

Tan, L. H., Liu, H. L., Perfetti, C. A., Spinks, J. A., Fox, P. T., \& Gao, J. H. (2001). The neural system underlying Chinese logograph reading. Neuroimage, 13, 836-846.

Tan, L. H., Spinks, J. A., Feng, C. M., Siok, W. T., Perfetti, C. A., Xiong, J., et al. (2003). Neural systems of second language reading are shaped by native language. Human Brain Mapping, 18, 158-166.

Tan, L. H., Spinks, J. A., Gao, J. H., Liu, H. L., Perfetti, C. A., Xiong, J. H., et al. (2000). Brain activation in the processing of Chinese characters and words: A functional MRI Study. Human Brain Mapping, 10, 1627.

Tan, L. H., Laird, A. R., Li, K., \& Fox, P. T. (2005). Neuroanatomical correlates of phonological processing of Chinese characters and alphabetic words: A meta-analysis. Human Brain Mapping, 25, 83-91.

Temple, E., Deutsch, G. K., Poldrack, R. A., Miller, S. L., Tallal, P., Merzenich, M. M., et al. (2003). Neural deficits in children with dyslexia ameliorated by behavioral remediation: Evidence from functional MRI. Proceedings of the National Academy of Science, 100, 2860-2865.

Thompson-Schill, S. L., D'esposito, M., Aguirre, G. K., \& Farah, M. J. (1997). Role of left inferior prefrontal cortex in retrieval of semantic knowledge: A reevaluation. Proceedings of the National Academy of Science, 94, 14792-14797.

Wagner, A. D., Koutstaal, W., Maril, A., Schacter, D. L., \& Buckner, R. L. (2000). Task-specific repetition priming in left inferior prefrontal cortex. Cerebral Cortex, 10, 1176-1184

Wagner, A. D., Pare-Blagoev, E. J., Clark, J., \& Poldrack, R. A. (2001). Recovering meaning: Left prefrontal cortex guides controlled semantic retrieval. Neuron, 31, 329-338.

Wang, H., Chang, R. B., \& Li, Y. S. (1985). Modern Chinese frequency dictionary. Beijing: Beijing Language University Press.

Wang, Y., Sereno, J. A., Jongman, A., \& Hirsch, J. (2003). fMRI evidence for cortical modification during learning of mandarin lexical tone. Journal of Cognitive Neuroscience, 15, 1019-1027. 
Worsley, K. J., Marrett, S., Neelin, P., Vandal, A. C., Friston, K. J., \& Evans, A. C. (1996). A unified approach for determining significant signals in images of cerebral activation. Human Brain Mapping, 4, 58-73.

Xue, G., Chen, C. S., Jin, Z., \& Dong, Q. (2006). Language experience shapes fusiform activation when processing a logographic artificial language: An fMRI training study. Neuroimage, 31, 1315-1326.
Xue, G., Dong, Q., Jin, Z., Zhang, L., \& Wang, Y. (2004). An fMRI study with semantic access in low proficiency second language learners. Neuroreport, $15,791-796$

Zhang, J. X., Zhuang, J., Ma, L. F., Yu, W., Peng, D. L., Ding, G. S., et al. (2004). Semantic processing of Chinese in left inferior prefrontal cortex studied with reversible words. Neuroimage, 23, 975-982. 